\title{
Experimental investigation of target and transducer effects on quantitative image reconstruction in photoacoustic tomography
}

John Gamelin, Andres Aguirre, Fei Huang, Anastasios Maurudis, Diego Castillo, et al.

John Gamelin, Andres Aguirre, Fei Huang, Anastasios Maurudis, Diego Castillo, Lihong V. Wang, Quing Zhu, "Experimental investigation of target and transducer effects on quantitative image reconstruction in photoacoustic tomography," Proc. SPIE 6437, Photons Plus Ultrasound: Imaging and Sensing 2007: The Eighth Conference on Biomedical Thermoacoustics, Optoacoustics, and Acousto-optics, 64371F (27 February 2007); doi: 10.1117/12.707245

SPIE. Event: SPIE BiOS, 2007, San Jose, California, United States 


\title{
Experimental Investigation of Target and Transducer Effects on Quantitative Image Reconstruction in Photoacoustic Tomography
}

\author{
John Gamelin ${ }^{* a}$, Andres Aguirre ${ }^{\mathrm{a}}$, Fei Huang ${ }^{\mathrm{a}}$, Anastasios Maurudis ${ }^{\mathrm{a}}$, Diego Castillo ${ }^{\mathrm{a}}$, Lihong V. Wang ${ }^{\mathrm{b}}$, \\ and Quing Zhu ${ }^{\mathrm{a}}$ \\ ${ }^{\mathrm{a}}$ Dept. of Electrical Engineering, University of Connecticut, Storrs-Mansfield, CT 06269 \\ ${ }^{b}$ Dept. of Biomedical Engineering at Washington University in St. Louis, St. Louis, MO 63130
}

\begin{abstract}
In principle, absorbed energy profiles can be exactly reconstructed from photoacoustic measurements on a closed surface. Clinical applications, however, involve compromises due to transducer focus, frequency characteristics, and incomplete measurement apertures. These tradeoffs introduce artifacts and errors in reconstructed absorption distributions that affect quantitative interpretations as well as qualitative contrast between features. The quantitative effects of target geometry, limited measurement surfaces, and bandpass transducer frequency response have been investigated using a ring transducer system designed for small animal imaging. The directionality of photoacoustic radiation is shown to increase with target aspect ratio, producing proportionate overestimates of absorption values for two-dimension apertures less than approximately 150 degrees. For all target geometries and orientations, mean absorption values approach the full view values for hemicircular measurement surfaces although the true spatial uniformity is recovered only with the complete surface. The bandpass transducer frequency spectrum produces a peaked amplitude response biased toward spatial features ranging from 1 to 8 times the system resolution. We discuss the implications of these results for design of clinical systems.
\end{abstract}

Keywords: Photoacoustic tomography, Limited view tomography, Clinical, Image reconstruction, Quantitative imaging, Array transducers

\section{INTRODUCTION}

Photoacoustic tomography has emerged as a promising biomedical imaging modality due to its ability to produce ultrasound-resolution images using optical contrast at depths up to 2 to $3 \mathrm{~cm}$ (Xu \& Wang, 2006). In comparison, well-established Diffusive Optical Tomography (DOT) techniques that also image optical contrast suffer from poor resolution of 1 to $3 \mathrm{~mm}$ due to intense scattering of light in the highly turbid in vivo tissue environment. DOT methods, however, have demonstrated a proven track record of quantitative determination of blood oxygenation, total hemoglobin concentrations, and absorption parameters in clinical trials (Culver et al., 2003; Pogue et al., 2001; Tromberg et al., 2000; Zhu et al., 2005). The success of diffusive approaches has leveraged heavily from developments of scattering models, source and detector instrumentation, and imaging algorithms over the past decade. Photoacoustic tomography is rapidly progressing from qualitative imaging to addressing the fundamental technological issues affecting quantitative measurements (Cox, Arridge, \& Beard, 2006; Yuan \& Jiang, 2006; Zemp, Li, \& Wang, 2006)

The broadband, coherent, and three-dimensional nature of photoacoustic generation presents several challenges for accurate reconstruction of absolute absorbed energy profiles. First, the spectrum of emitted acoustic frequencies is dependent upon both the illumination pulse and the absorption distribution. This is in contrast to ultrasound imaging where the response is determined by the narrowband transmitter excitation. Because most tissues exhibit spatial frequencies ranging from low-frequency background to high-frequency features such as blood vessels, the photoacoustic signals possess significant spectral energy outside the bandwidth of even state-of-the-art wideband transducers.

Photons Plus Ultrasound: Imaging and Sensing 2007: The Eighth Conference on Biomedical Thermoacoustics, Optoacoustics, and Acousto-optics, edited by Alexander A. Oraevsky, Lihong V. Wang, Proc. of SPIE Vol. 6437, 64371F, (2007) - 1605-7422/07/\$18 - doi: 10.1117/12.707245 
Second, the (nearly) simultaneous excitation of the tissue due to the short $(<15 \mathrm{~ns})$ optical pulses and the high speed of light produce phase-coherent acoustic signals. The limited scattering at ultrasonic wavelengths preserves this coherency throughout propagation to the detectors. As a result, absorption features extending greater than a half wavelength act as phased antennas and can exhibit very directional radiation patterns. This concentration of photoacoustic energy can be oriented out of the field of view and limit visibility. Reconstruction errors, both qualitative and quantitative, also occur when the energy is oriented within the field of view. Transducer elements will capture a greater fraction of highly directional photoacoustic emission from flat, specular interfaces relative to rounded isotropic features that radiate uniformly. As a result relative contrast and quantitative imaging can be biased toward highly oriented absorption profiles.

Third, practical considerations limit the achievable measurement surface for capturing the photoacoustic signals. Clinical applications, for example, provide limited access due to physical constraints (e.g. interfering tissue) or limited penetration of the optical signals due to absorption and scattering. Furthermore, cost and size can further restrict the measurement aperture. Focused transducers are often employed to improve the detection sensitivity and the resulting acoustic response profile additionally limits the measurement volume. The combination of these factors, collectively known as the limited-view problem, limits the available photoacoustic signals for imaging and introduces geometric and quantitative artifacts. The impacts of limited measurement apertures on photoacoustic reconstruction have been extensively studied in recent years (Anastasio \& Zhang, 2006; Patch, 2004; Pan, Zou, \& Anastasio, 2003; $\mathrm{Xu}$, Wang, Ambartsoumian, \& Kuchment, 2004) with emphasis upon identification of sufficiency criteria and simulation results. These studies, however, considered only square and circular targets and provided limited experimental validation. Furthermore, the effect of transducer frequency response on quantitative reconstruction was not examined.

For quantitative photoacoustic tomography to become a reality, each of these challenges must be understood and technological solutions identified. The aim of this work is to quantify the effects of each of these parameters as a first step towards addressing the critical issues for development of a clinical photoacoustic system. We experimentally investigate the effect of target and transducer effects in quantitative photoacoustic imaging. We examine the impact of absorption geometry and orientation in determining the radiation profiles and the resulting reconstructions using a ring transducer system designed for small animal experimentation. The influence of transducer frequency response is also evaluated and the variations with feature size are correlated with an analytic model of the photoacoustic signal. The implications of these observations for clinical system and experimental design are addressed.

\section{MATERIALS AND METHODS}

\section{Experimental Setup}

Figure 1 depicts a picture of the experimental configuration. A Ti:Sapphire (Symphotics TII, LS-2134) laser optically pumped with a Q-switched Nd:YAG laser (Symphotics-TII, LS-2122) delivered 8-12 ns pulses at $15 \mathrm{~Hz}$ and $780 \mathrm{~nm}$ wavelength. The beam was diverged with a plano-concave lens and homogenized by a circular profile engineered diffuser (ED1-S20, ThorLabs, Newton, NJ) to produce a uniform illumination of approximately $50 \mathrm{~mm}$ in diameter at the sample. The radiance at the sample was below $1 \mathrm{~mJ} / \mathrm{cm}^{2}$ for all experiments. The laser light was positioned at the center of curvature of a 90 -degree annular transducer submerged in a 50-gallon water tank. The light was incident orthogonal to the ring transducer imaging plane for maximum uniformity. 


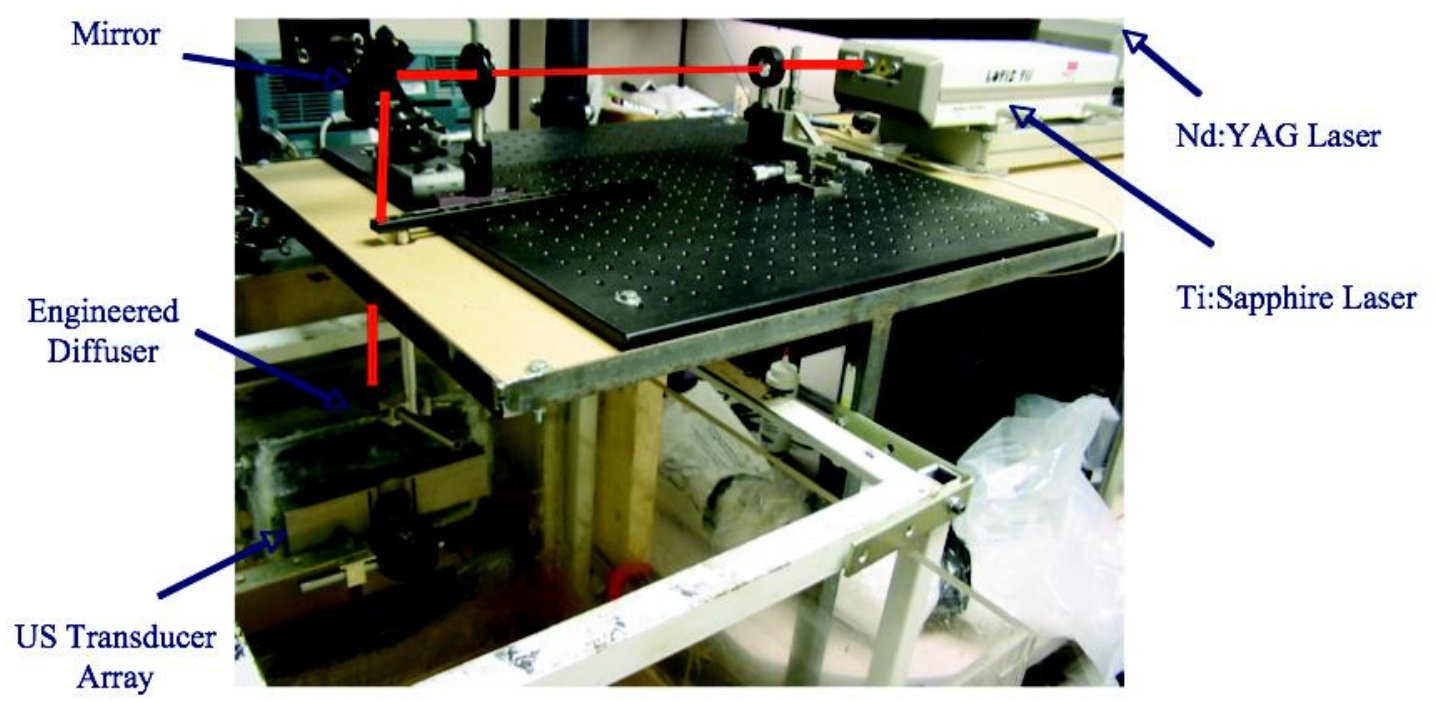

Figure 1. Experimental Setup. A Nd:YAG pumped Ti:Sapphire laser operating at $780 \mathbf{~ n m}$ and $\mathbf{1 5}$ $\mathrm{mJ}$ is expanded, turned with a mirror, and diffused to a diameter of over $50 \mathrm{~mm}$. The beam illuminates the samples orthogonal to the ring transducer array. A rotational stages rotates the samples in 90 degree increments to emulate a full 512 element array.

Details of the transducer design and characterization have been described elsewhere (Maurudis et al., 2006). In brief, the transducer consists of 128 elements arranged along a $90^{\circ}$ arc with a $25 \mathrm{~mm}$ center of curvature. The array was designed as a building block for a 512 element closed ring system. The array was custom fabricated by Imasonic, Inc. (Besançon, France) using piezocomposite technology for high sensitivity and signal-to-noise ratio. The center frequency of the array was $5 \mathrm{MHz}$ with a reception bandwidth of greater than $60 \%$. Individual elements featured an elevation height of $10 \mathrm{~mm}$ with an azimuthal pitch of one wavelength $(0.308 \mathrm{~mm})$ and kerf of $0.1 \mathrm{~mm}$. The array elements were shaped in the elevation direction to produce an arc-shaped focus at $19 \mathrm{~mm}$ from the transducer without the distortions and loss of sensitivity encountered with external acoustic lens materials. In the full 512-element configuration this focus results in a uniform central imaging region of approximately $16 \mathrm{~mm}$ in diameter.

The photoacoustic signals from each element were individually amplified $60 \mathrm{~dB}$ and multiplexed into 16 data acquisition channels sampling at $40 \mathrm{MHz}$ with 12-bit precision. Due to the multiplexing, eight laser firings were required to generate a single 128-channel capture. The data was DMA transferred to RAM and subsequently disk for post-processing. In all experiments presented here 32 data averages were performed although adequate signal-to-noise ratio was achieved for high-quality single-capture imaging.

All samples were mounted on a rotary stage positioned at the radius of curvature that was turned in $90^{\circ}$ increments to emulate the response of a full ring. The temperature was continuously monitored with a digital thermometer for precise determination of the sound speed. Without corrections using this measurement, temperature variations throughout the duration of experimentation produced registration errors of up to 500 microns in $360^{\circ}$ tomographic imaging. All images were reconstructed using the exact backprojection algorithm of Xu and Wang (Xu \& Wang, 2005). The solid-angle weighting factor of this formulation, designed to minimize errors in the limited view tomography, was alternately incorporated and eliminated in the reconstructions. By setting the weighting factor to unity the absolute buildup of photoacoustic signals with number of array elements could be investigated and the effects of the solid angle factor independently evaluated. The implementation of the algorithm included Wiener inverse filtering of the measured data to account for the measured transducer impulse response while limiting the effects of noise in the low and high frequency regions. In addition, theoretically calculated corrections for the directivity of individual elements, assuming a rectangular element profile, were incorporated. 


\section{Investigation of target geometry and orientation relative to measurement aperture}

To examine the effects of target geometry and orientation, India ink-filled polyethylene tubes with an inner diameter of 580 microns were used as contrast targets. The tubes, with lengths ranging from $1.5 \mathrm{~mm}$ to 10 $\mathrm{mm}$, were hot glued onto the tips of $1 \mathrm{~mm}$ plastic optical fiber for mounting. The corresponding aspect ratios of these structures were $3: 1$ to $18: 1$. The tubes were oriented parallel to the transducer face for the quarter ring configuration as depicted in Figure 2(a). A vertically mounted (i.e. parallel to the illumination) 80 micron black nylon thread served as an isotropic source with 1:1 aspect ratio. The radiation patterns for all targets were evaluated using the dominant far-field term of the backprojection formula: $-\left.t \frac{\partial p}{\partial t}\right|_{t=R c}$ for

all elements as the samples were rotated $360^{\circ}$. In this formula " $\mathrm{t}$ " is the time equal to the product of the radius of curvature $(\mathrm{R})$ and sound speed " $\mathrm{c}$ " and " $\mathrm{p}$ " is the detected pressure signal. The radiation amplitude versus direction was plotted to yield the photoacoustic radiation pattern as a function of the target aspect ratio.

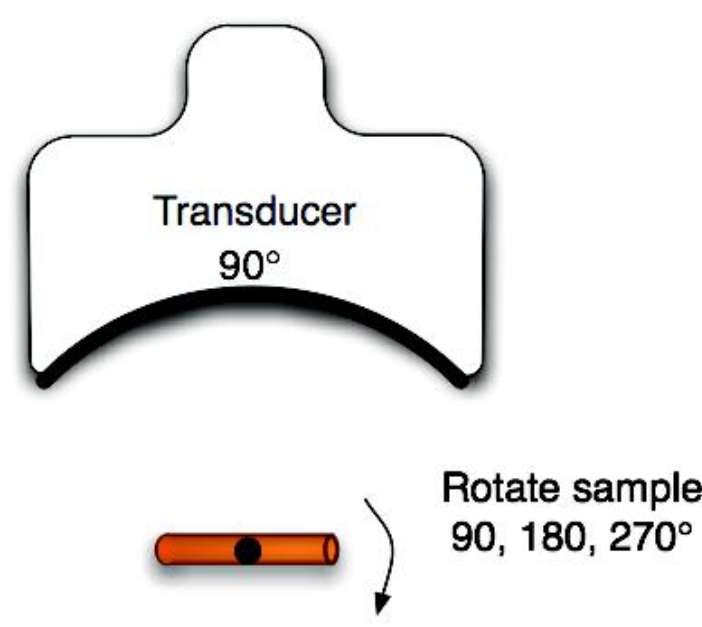

(a)

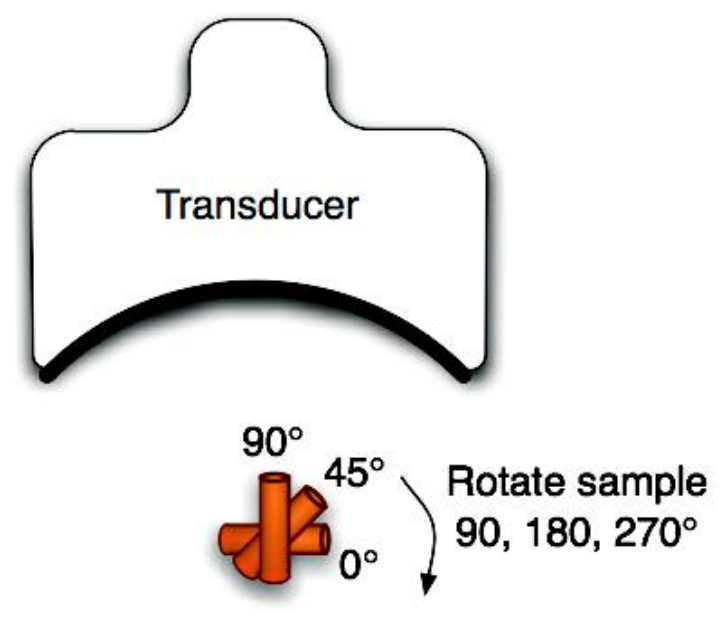

(b)

Figure 2. Experimental configuration for investigation of target orientation and aspect ratio effects. (a) An 80 micron black thread and India ink-filled tubes of 580 micron diameter and lengths from 1.5 to $10 \mathrm{~mm}$ served as targets with aspect ratios of $1: 1$ to $18: 1$. (b) A $5.5 \mathrm{~mm}, 580$ micron inner diameter ink-filled tube was oriented at 0,45 , and $90^{\circ}$ with respect to the transducer. For both experiments, the samples were rotated in 90 degree increments with the indicated position in (a) serving as the 90 degree reference for the radiation patterns.

In a second set of experiments examining the effect of target orientation, the $5.5 \mathrm{~mm}$ long, 580 micron inner diameter tube was fixed at the elevation focus in the quarter circle configuration as depicted in Figure 2(b). The tube was orientation parallel, perpendicular, and at 45 degrees to the azimuthal axis. The reconstructed images were quantitatively analyzed for measurement apertures ranging from 32 to 512 elements. As described earlier, the analysis considered the reconstructions with and without the solid angle weighting factor.

\section{Investigation of target size}

The impulse response of the transducer array was measured by illumination of the element faces with isotropically scattered light. Figure 3 depicts the amplitude frequency response along with the theoretical 
spectra for uniformly absorbing spheres with radii $\left(r_{s}\right)$ of 1 and $5 \mathrm{~mm}$. The center frequency $\left(0.33 \mathrm{c} / r_{s}\right)$ and $3 \mathrm{~dB}$ bandwidth $\left(0.35 \mathrm{c} / \mathrm{r}_{s}\right)$ of the main lobe of the $2 \mathrm{~mm}$ diameter sphere match the transducer response well. In contrast, the $10 \mathrm{~mm}$ diameter sphere possesses a narrower spectrum located near the lowfrequency edge of the transducer response. As a result the system should exhibit an optimum sensitivity for objects with dimensions near 200 to 500 microns.

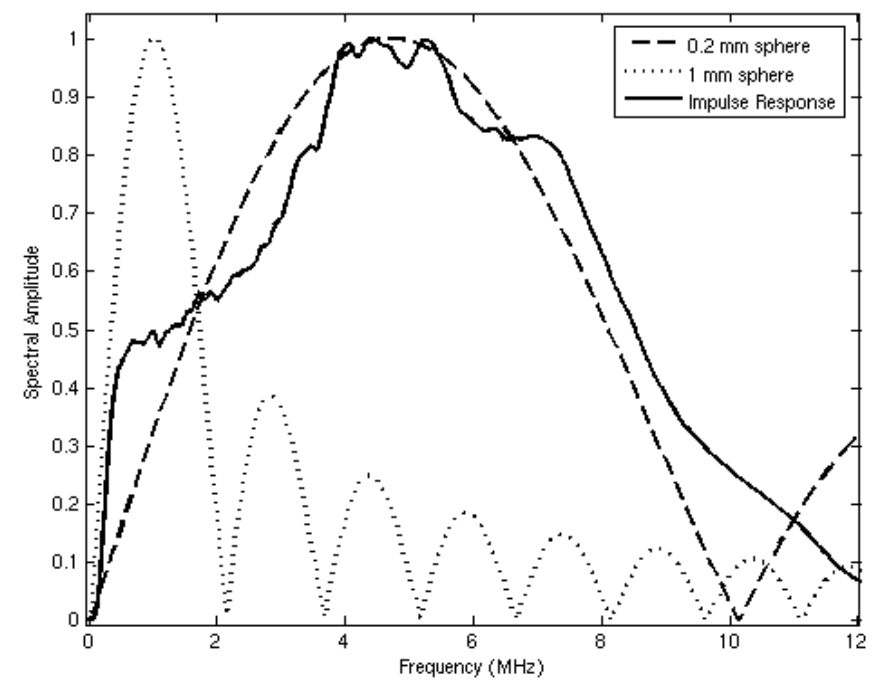

Figure 3. Comparison of transducer element impulse response (solid) and the frequency spectrums of solid absorbing spheres with diameters of 0.2 and $1.0 \mathrm{~mm}$. The transducer response was measured by illumination of the transducer surface diffusely with the laser beam.

In order to quantitatively evaluate the effect of target size on the reconstructed absorption value, a $25 \mathrm{~mm}$ uniform ink-dyed gelatin phantom was employed. Cylindrical disk targets with diameters ranging from 200 micron to $6 \mathrm{~mm}$ were produced by selective illumination through an absorbing mask as illustrated in Figure 4. The circular absorption profile was located near the ring center of curvature to minimize interelement variations due to the elevation focus and images were based upon 128 elements (quarter ring). The mean value and normalized standard deviation (= standard deviation/mean) of the disk absorption profile were calculated from the backprojection reconstructions with solid angle weighting. Inverse filtering of the impulse response is often used to equalize the phase and amplitude characteristics of the transducer elements. The effects of this signal processing in modifying the size response were investigated by incorporated phase-only and full phase/amplitude inverse filtering. The filter parameters were chosen to limit inverse filtering to components of the spectrum with frequencies between $400 \mathrm{kHz}$ and $10 \mathrm{MHz}$ to reduce amplification of noise. 


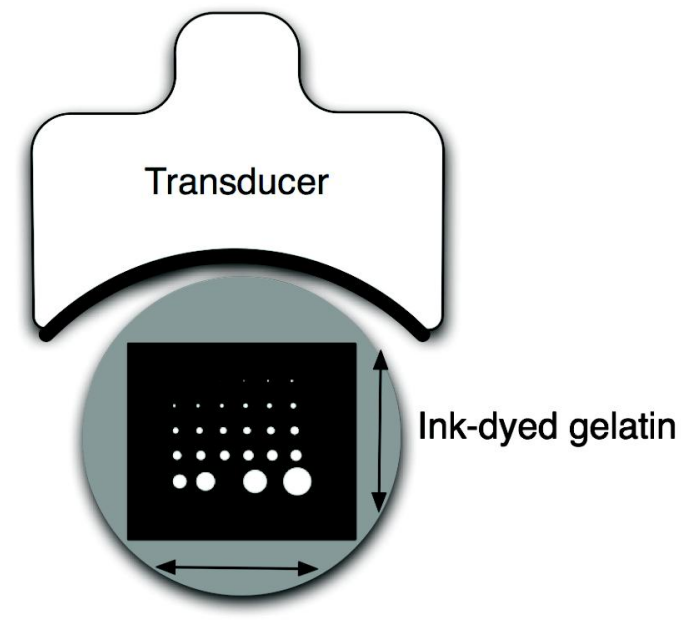

Figure 4. Experimental configuration for evaluation of target size effects. Selective illumination of a uniform ink-dyed gelatin cylinder produced absorption disks with diameters ranging from 0.2 to

$6 \mathrm{~mm}$. The mask was translated to maintain the same position within the transducer field.

\section{RESULTS AND DISCUSSION}

\section{Effects of target aspect ratio and orientation}

Figure 5 depicts the photoacoustic radiation patterns for the 80 micron black thread and ink tubes. The $90^{\circ}$ position corresponds to the origin of the measurement aperture for the following reconstructions. The radiation is isotropically uniform for the cylindrical thread with progressive directionality with increased aspect ratio. The asymmetry in the side lobes oriented along $0^{\circ}$ and $180^{\circ}$ are due to acoustic interference from the mounting fiber and glue. For ratios greater than 5:1 the photoacoustic energy is concentrated in a narrow beam perpendicular to the tube surface $\left(90^{\circ}\right.$ and $\left.270^{\circ}\right)$ along its length similar to a dipole antenna. This high directionality results in a small number of transducers with a significant contribution to the reconstruction.

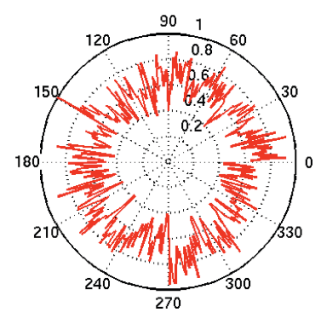

$1: 1$

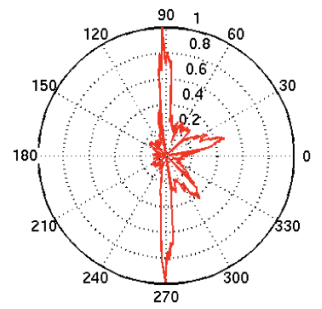

10:1

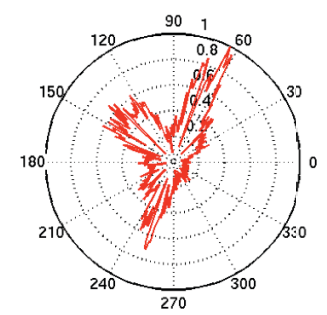

$5: 1$

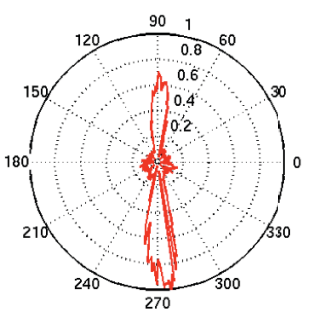

18:1

Figure 5. Measured radiation patterns for 80 micron black thread (1:1) and 580 micron tubes with lengths corresponding to aspect ratios of $3: 1$ to $18: 1$. 
Figure 6 depicts the mean reconstructed values of absorption within the tubes normalized to their respective volumes as a function of the two-dimensional (2D) measurement aperture. For this figure the solid angle weighting factor was set to unity to display the unnormalized accumulation of photoacoustic energy with the number of transducer elements. Consistent with the radiation patterns, the accumulated backprojection absorption terms increase linearly and monotonically with the 2D solid angle for the cylindrical thread. With increasing aspect ratio (length) the energy contributions occur predominantly at the ends of the scan range resulting in a sigmoidal characteristic.

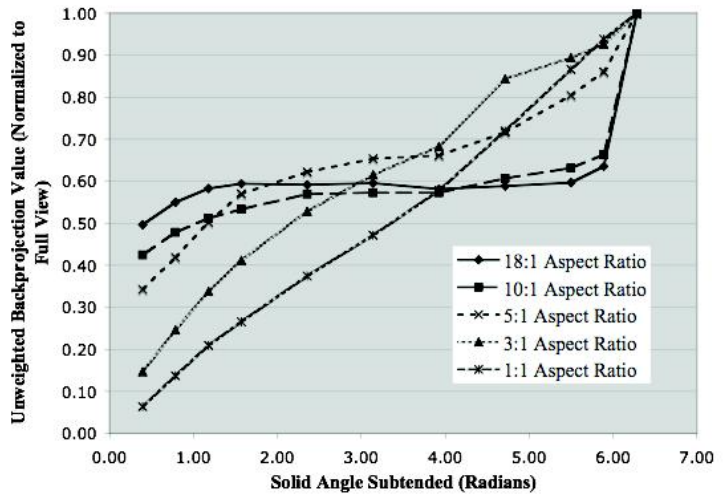

(a)

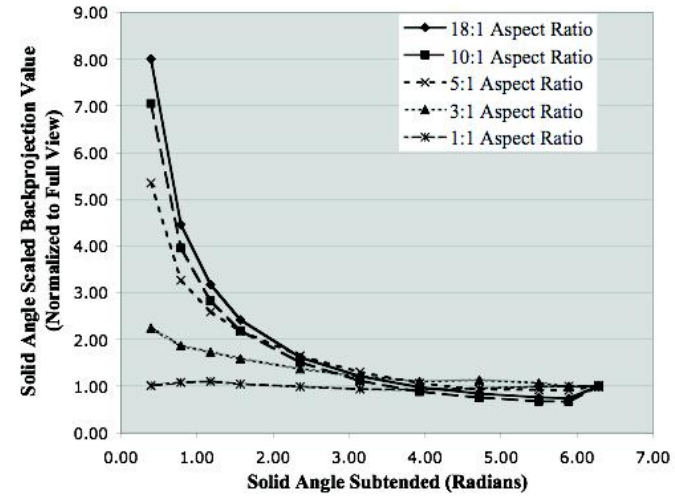

(b)

Figure 6. Plot of estimated absorption values for black thread and ink tubes without (a) and with (b) solid-angle normalization using the exact backprojection algorithm of $\mathrm{Xu}$ and Wang.

Incorporation of the solid angle weighting factor produces the monotonically decreasing estimated absorption shown in Figure 6. All absorption values have been normalized to the full ( $2 \pi$ radians) value. In agreement with earlier simulation studies (Xu, Wang, Ambartsoumian, \& Kuchment, 2004), the reconstruction error asymptotically diminishes as the measurement aperture approaches half-view $\left(180^{\circ}\right)$. For smaller apertures the reconstruction overestimates the true absorption value for all sample geometries. The results indicate that clinical applications (e.g. breast or epithelial tissues) that limit the aperture to approximately 150 degrees or less can result in absorption overestimates of greater than 30 to $60 \%$. The amount of overestimation will depend upon the solid angle subtended by the feature of interest and therefore will vary across the imaging field of view. Furthermore, the observations indicate that the coherent and directional radiation patterns produce a strong dependence upon the geometry of the absorption profile and therefore cannot be calibrated out. The implication of this fact is that features within the imaging field will produce differing contrast and reconstructed absorption values that can vary by up to $30 \%$ or more for measurement surfaces less than $180^{\circ}$. Reductions in this amount of variation may be possible through enhanced model-based reconstruction algorithms that can account for the acoustic propagation.

The highly directional radiation of high aspect ratio absorption features can concentrate the photoacoustic energy to positions near or outside the imaging field. Figure 7 depicts the measured mean absorption value for a $5.5 \mathrm{~mm}$ long ink-filled tube oriented parallel, perpendicular, and at $45^{\circ}$ to the azimuthal center of the transducer. The estimated absorption values shown in Figure 7(a) obtained without solid angle weighting illustrate the more progressive increase in captured photoacoustic energy with the non-parallel orientations. Incorporation of the solid angle weighting (Figure $7(b)$ ) reveals that the gradual increase in captured photoacoustic energy with measurement aperture results in reduced sensitivity to the size of the total transducer surface. In contrast, the high aspect ratio feature oriented such that only one direction intercepts the measurement surface exhibits a high sensitivity to the orientation and extent of the transducer surface. 


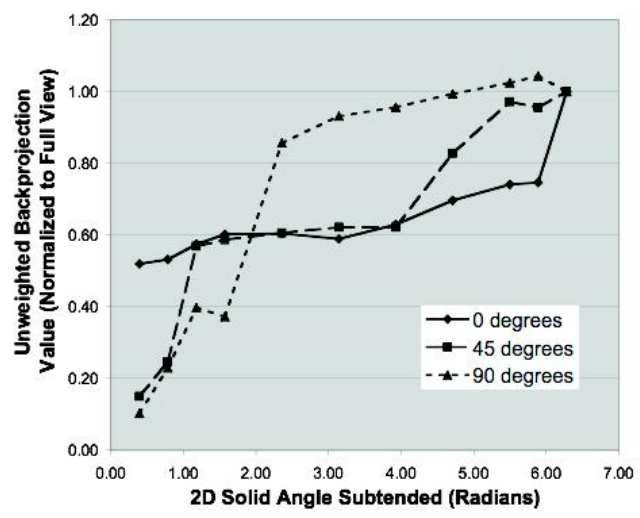

(a)

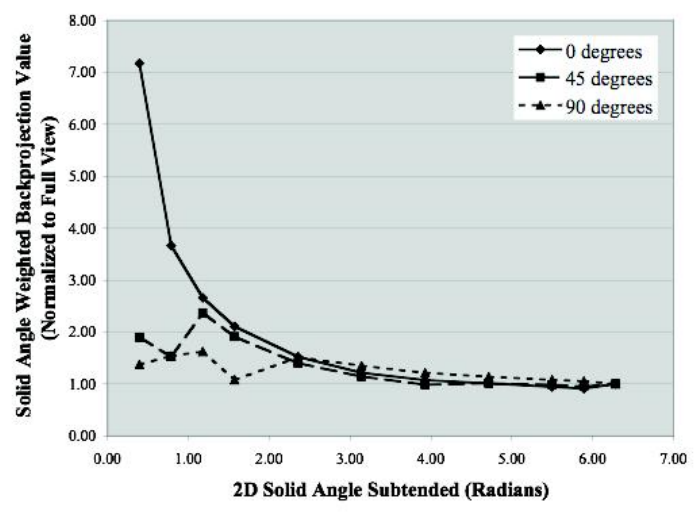

(b)

Figure 7. Graphs of estimated absorption values for $5.5 \mathrm{~mm}$ long ink tube oriented at different angles with respect to the transducer with (a) and without (b) the solid angle normalization factor using the backprojection algorithm of $\mathrm{Xu}$ and Wang.

\section{Effects of feature size}

Figure 8(a) shows the normalized mean absorption value for cylindrical absorption profiles with diameters ranging from 200 microns to $6 \mathrm{~mm}$. The data have been normalized to the largest estimated absorption. The graph indicates a response peaked at 300-400 micron diameter due to the bandpass transducer response with a sharp rolloff for smaller and larger sizes. The optimum diameter is slightly larger than that suggested by Figure 3 (200 micron), perhaps due to contributions for the higher frequency lobes of the photoacoustic signal and from differences in the photoacoustic frequency spectrum for disk versus spherical sources. A $3 \mathrm{~dB}$ criterion (0.7071) for maximum amplitude variations translates to a target size range of $\sim 100$ to 800 microns, less than a ten-fold range biased toward the system resolution of 160 microns. Although this result is specific to our system, state of the art wideband transducers possess bandwidths of $60-80 \%$ and should exhibit similar amplitude responses. Separation of the phase (solid) and amplitude/phase (dashed) inverse-filtering effects indicates some improvement in relative sensitivity for the larger diameters by equalization. Note that the curves are separately normalized with respect to feature size as the amplitude equalization produces higher absolute reconstructed values.

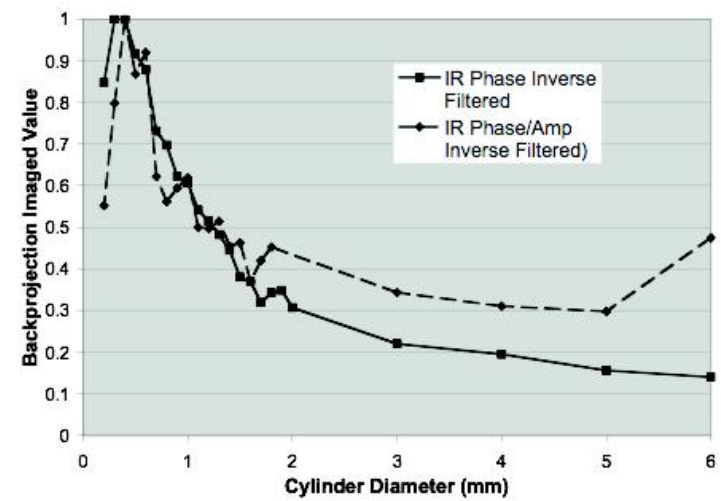

(a)

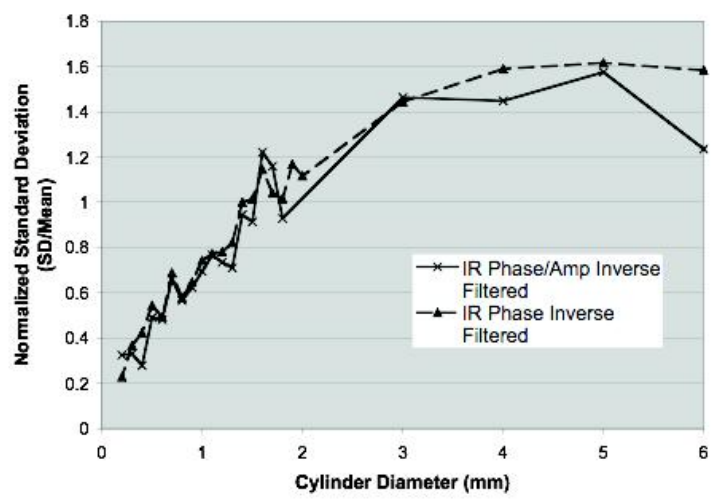

(b)

Figure 8. Graphs of mean value (a) and normalized standard deviation (b) for absorbing gelatin cylinders with diameters from 0.2 to $6 \mathrm{~mm}$. The solid curves designate results using inverse filtering with the phase of the measured impulse response and dashed curves correspond to results with both amplitude and phase inverse filtering. 
Figure 8(b) depicts the corresponding variations in uniformity for the absorption disks. For both phase and phase/amplitude inverse filtering the normalized standard deviation declines monotonically with feature size. This improvement reflects the better matching of the frequency spectrum to the bandpass transducer response and concomitant fidelity in reproducing the uniform absorption profile.

\section{Implications for quantitative clinical system design}

The experimental results highlight the strong dependences of quantitative reconstructed values on target geometry, orientation, and size, even when possessing equal absorption properties. For all target geometries and orientations, increasing the measurement aperture improves the reconstruction accuracy. However, although the errors in mean estimated absorption reduce asymptotically beyond a hemicircular or hemispherical measurement surface, feature details can still be exaggerated and lead to false conclusions regarding the uniformity or peak absorption value. Figure 9 illustrates this behavior with the reconstructed profiles for a 580 micron ink-filled tube using apertures of 128 (quarter circle), 256 (half-circle), and 512 (full circle) transducers. This case corresponds to the 10:1 aspect ratio target of Figure 6 . The images demonstrate that while the mean absorption differs by less than $25 \%$ for 256 elements, the homogeneity of the absorption within the tube is not fully realized until 512 elements capture the radiation in all planar directions. Features larger than the dimensions of optimum sensitivity are particularly vulnerable to imaging artifacts with limited views as the second half of a full scan can provide resolution of complementary interfaces whose profile is distorted due to the finite transducer frequency response.
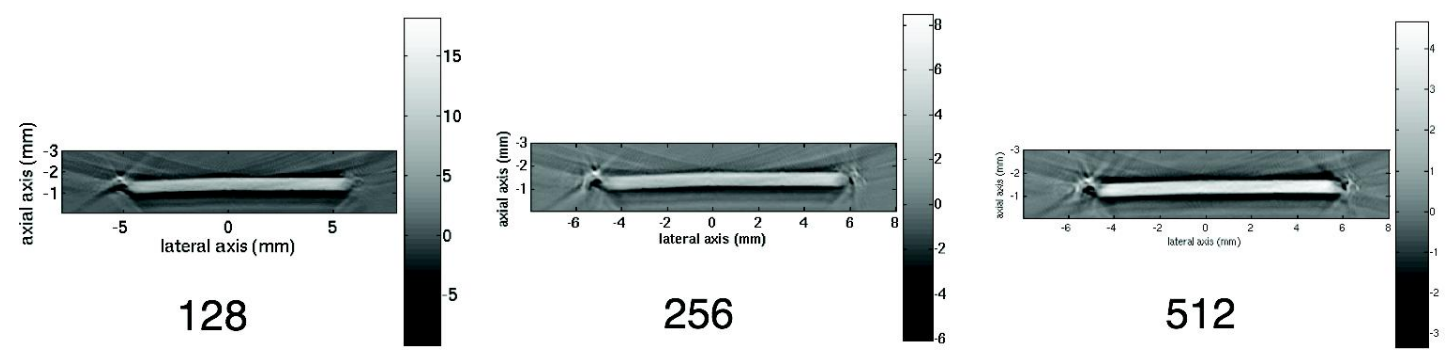

Figure 9. Images of an $1 \mathrm{~cm}$ ink-filled tube of 580 micron diameter reconstructed with 128, 256, and 512 elements. The absorption values are overestimated with the partial views and the uniformity is not recognized except in the full view (512).

Although larger measurement apertures can minimize the effect of sample feature orientation and geometry, feature size variations present a greater technological challenge. Figure 6 and Figure 7 illustrated that the variations due to apertures is approximately a factor of two whereas sensitivity differences of five or more are possible for realistic targets. As a result, center frequencies of transducers must be carefully chosen so that the resolution is approximately five to ten times the largest feature to be imaged quantitatively to reduce variations to less than $30 \%$ for state-of-the-art wideband transducers. Uniform performance for a wider range of dimensions will require ultra wideband transducers. Oraevsky (Oraevsky \& Karabutov, 2000) has studied the tradeoff between sensitivity and bandwidth for various piezoelectric materials in photoacoustic applications. In practice, as with ultrasound imaging, multiple transducer heads with differing frequency response characteristics may be required to improve imaging accuracy. The variations with feature size will still be present for each transducer, however, complicating interpretations of contrast and quantitative parameters.

\section{CONCLUSION}

In this work we experimentally investigated the effects of geometry, orientation, and size of absorbing targets upon quantitative photoacoustic reconstruction. The radiation patterns of structures were shown to exhibit directionality in direct proportion to the aspect ratio of the absorbing targets. As a result, overestimates of the absorption coefficient occur for limited view apertures and most of the acoustic energy 
can be directed outside of the measurement surface even when the target is within the nominal imaging field of view. The finite bandwidth of wideband transducers was also demonstrated to introduce a sizedependent quantitative reconstruction sensitivity that limits accuracy for features more than approximately 5 to 10 times the system resolution. These results further emphasize the need for measurement apertures greater than $\sim 120^{\circ}$ and either multiple or ultra wideband transducers matched to the clinical application.

\section{ACKNOWLEDGEMENTS}

We acknowledge partial support from NIH grants NIH R01 NS46214 and NIH R01EB002136. The author can be contacted at (860) 486-3673 or via e-mail at jkg@engr.uconn.edu.

\section{REFERENCES}

Cox, B. T., Arridge, S., \& Beard, P. (2006). Quantitative Photoacoustic Image Reconstruction for Molecular Imaging. Proceedings of SPIE, 6086, 60861M-6081:9.

Culver, J. P., Choe, R., Holboke, M., Zubkov, L., Durduran, T., Slemp, A., et al. (2003). Threedimensional diffuse optical tomography in the parallel plane transmission geometry: Evaluation of a hybrid frequency domain/continuous wave clinical system for breat imaging. Med. Phys, 30(2), 235-247.

Anastasio, M. A. \& Zhang, J. (2006). Image reconstruction in photoacoustic tomography with truncated cylindrical measurement apertures. Proceedings of SPIE, 6086, 608610$1: 608610-7$.

Maurudis, A., Huang, F., Guo, P., Yan, S., Castillo, D., Wang, L. V., et al. (2006). A photoacoustic imaging system employing a curved-phased ultrasonic array and parallel electronics. Proceedings of SPIE, 6086, 60861Q-601:10.

Oraevsky, A. \& Karabutov, A. (2000). Ultimate Sensitivity of Time-Resolved Opto-Acoustic Detection. Proceedings of SPIE, 3916, 228-239.

Patch, S. K. (2004). Thermoacoustic tomography - consistency conditions and the partial scan problem. PHYSICS IN MEDICINE AND BIOLOGY, 49(11), 2305-2315.

Pogue, B. W., Poplack, S. P., McBride, T. O., Wells, W. A., Osterman, K., Osterberg, U., et al. (2001). Quantitative hemoglobin tomography with diffuse near-infrared spectroscopy: pilot results in the breast. Radiology, 218, 261-266.

Tromberg, B., Shah, N., Lanning, R., Cerussi, A., Espinoza, G., Pham, T. H., et al. (2000). Noninvasive in vivo characterization of breast tumors using photon migration spectroscopy. Neoplasia, 2(1:2), 26-40.

Pan, X., Zou, Y., \& Anastasio, M. A. (2003). Data redundancy and reduced scan reconstruction in reflectivity tomography. IEEE Transactions on Image Processing, 12(7), 784-795.

Xu, M. \& Wang, L. V. (2005). Universal back-projection algorithm for photoacoustic computed tomography. Phys Rev E Stat Nonlin Soft Matter Phys, 71(1 Pt 2), 016706.

$\mathrm{Xu}$, M. H. \& Wang, L. H. V. (2006). Photoacoustic imaging in biomedicine. REVIEW OF SCIENTIFIC INSTRUMENTS, 77(4), 041101.

Xu, Y., Wang, L. V., Ambartsoumian, G., \& Kuchment, P. (2004). Reconstructions in limitedview thermoacoustic tomography. MEDICAL PHYSICS, 31(4), 724-733.

Yuan, Z. \& Jiang, H. B. (2006). Quantitative photoacoustic tomography: Recovery of optical absorption coefficient maps of heterogeneous media. APPLIED PHYSICS LETTERS, 88(23), 231101.

Zemp, R. J., Li, L., \& Wang, L. V. (2006). Fundamental Considerations for Multiwavelength Photoacoustic Molecular Imaging. Proceedings of SPIE, 6086, 60861L-6081:9.

Zhu, Q., Cronin, E. B., Currier, A. A., Vine, H. S., Huang, M., Chen, N., et al. (2005). Benign versus malignant breast masses: optical differentiation with US-guided optical imaging reconstruction. Radiology, 237(1), 57-66. 